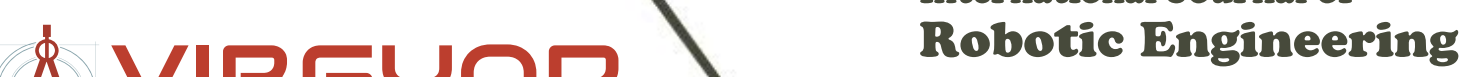

ISSN: 2631-5106

\section{Application of Social Robots for Symptom Control in Institutionalized Elderly Patients with Dementia}

\author{
Ana Nunes Barata ${ }^{1 *}$, Henrique G Martins ${ }^{2}$ and Rita Veloso Mendes ${ }^{3,4}$ \\ ${ }^{1}$ UCSP Buraca, Portugal \\ ${ }^{2}$ Shared Services of the Ministry of Health, Portugal \\ ${ }^{3}$ Department of Planning and Organizational Development, Shared Services of the Ministry of Health, Portugal \\ ${ }^{4}$ Public Health Research Center, National School of Public Health, University of Lisbon, Portugal
}

\begin{abstract}
Context: World population is rapidly growing older and the need for long term care is rising, namely in conditions such as dementia. Complementary therapies have shown to be beneficial for symptom management in dementia, namely the animal-assisted therapy (AAT). Due to limitations for the use of animals in institutions, social robots with zoomorphic traits have been created as alternative and some models have already shown to have similar effects to AAT.

Objectives: Understanding how a widely commercialized pet robot designed for children, as a context modifier, can stimulate social interaction and improve quality of life of institutionalized patients with dementia.

Methods: Qualitative study on six participants with cognitive impairment that were randomly selected at a senior living community in Portugal. Cognition was assessed with the Short Portable Mental Status Questionnaire. Participants were exposed to a 2012 model of Furby ${ }^{\odot}$ during one week and the interaction was recorded.

Results: Findings showed that Furby ${ }^{\odot}$ had effects on people with moderate and severe dementia. Nearly all participants smiled while interacting with the robot and they moved in order to comply with simple tasks. It also facilitated spontaneous dialogue as well as promoted interpretative thinking.

Conclusion: Furby ${ }^{\odot}$, a widely commercialized robot designed for children, has stimulated positive behaviors in elderly patients with dementia, who are living at an institution. It has shown to be a means to facilitate the expression of positive emotions as well as a stimulator for motor and cognitive activity. However, Furby ${ }^{\odot}$ has shown to have some limitations in terms of its design, namely in patients that were bedridden and in terms of controlling its personality and sounds.
\end{abstract}

\section{Keywords}

Robotics, Dementia, Aged 80 and over, Behavioral symptoms, Complementary therapies, Health facilities

\section{Introduction}

Robotics has developed into an educational and technological field. Efforts have been made since then to create a robot that is able to actively interact with the human being on a social level.
The robots that have been developed until now took their inspiration on known life forms that can be found in our planet. Therefore, their design is fundamentally anthropomorphic or zoomorphic. Due to the galloping developments in technology, it has been possible to

*Corresponding author: Ana Nunes Barata, Family Doctor, MSc in Palliative and Hospice Care, student of the Postgraduate Degree in Geriatrics, UCSP Buraca, R. Luís de Camões 5, 2720-344 Buraca, Amadora, Portugal, Tel: +351-21-472-5530, E-mail: ana.barata@csreboleira.min-saude.pt

Received: January 18, 2017: Accepted: March 27, 2017: Published: March 29, 2017

Copyright: (c) 2017 Barata AN, et al. This is an open-access article distributed under the terms of the Creative Commons Attribution License, which permits unrestricted use, distribution, and reproduction in any medium, provided the original author and source are credited. 
open up new horizons and, consequently, find new fields where they may be applied. This includes the possibility of doing research on human-robot interactions such as studying robots as facilitators for social interaction, as well as to learn of their direct impact on the cognitive function and the behavior of human beings [1-4].

The current demographics show that the world's population is rapidly growing older, mainly because of the global drop of birth rates. Comparing data from fifty years ago, populations live on average twenty years longer, what increases the prevalence of no communicable diseases. As such, the need for long term care rises exponentially, as many elderly lose the ability to live independently and look after themselves [5].

Dementia is known to be a disease where mainly the cognitive function is affected. Therefore, it has a direct impact on the patient's communication skills, which makes them more prone to a depressive humor. Consequently, patients tend to isolate themselves, developing feelings of loneliness which will further aggravate their mental well-being. Considering that it is a progressive and life-limiting disorder, there has been an increased use of complementary therapies in order to improve symptom management (such as agitation and depression) and to attempt to slow down the progression of the disease by stimulating the cognitive function and motor activities. Robotics has found here an opportunity for its implementation as a means to alleviate patients' symptoms and boost cognitive and motor activities [6].

Complementary therapies wish to stimulate, promote social interaction and the change of routines in patients, being some of the most frequently used forms of complementary therapy the animal-assisted therapy, music therapy and art therapy. Animal-assisted therapy is one of the therapies shown to have one of the most remarkable effects [7]. This can be understood thanks to the millennial relationship between human beings and other animals-the first fossil evidence showing an association between Homo erectus and a canine-like species dates from half a million years ago [8].

There are two theories to justify this relationship: the biophilia hypothesis and the social support hypotheses. The first justifies that the human being has an inapt tendency to take care and to feel attraction for other animals or living beings. The social support hypotheses claims that pets are per se already a social support [8].

However, it's difficult to study the therapeutic interventions with a pet due to hygienic, allergenic or institutional reasons. Progress has been made to study the effects of complementary therapy with zoomorphic robots which have shown to have similar effects to animal-assisted therapy, such as increased ability for social interaction, decreased levels of agitation, aggressiveness and depressive symptoms [9-13]. These results have been measured by analytical tests, imaging and electrophysiological monitoring methods [1416]. Results have also been observed in terms of increased nutritional intake and less need for medication and medical visits. In terms of effects on the cognitive function, the evidence is still not clear.

In this context, there is the possibility to do research with social robots that take on the form of animals. There are many different models available including the mass-commercialized robot known as Furby ${ }^{\circ}$. This robot was developed for children and it was created to interact with children by touch, voice and movement [17]. So, it's not farfetched to imagine its use in elderly people that may be weakened due to the effects of ageing or a disease.

Furby $^{\oplus}$ have some emotional features that are mostly expressed by sound and movements (e.g.: its voice gets louder when it's happier. There is also an increase of ear and torso movements). The robot has few facial expressions and there is an attempt to express them through the LCD eyes.

With the support of the Innovation and Research Area of the Department of Planning and Organizational Development of the Shared Services of Ministry of Health, a project with Furby ${ }^{\odot}$ was developed. We designed an observation in order to understand how a widely commercialized pet robot, developed for children, could stimulate, as a context modifier, social interaction and improve the quality of life of institutionalized patients with dementia. The observation ran at a senior living community in Sesimbra, Portugal and the participants were selected randomly (all participants had some level of cognitive impairment). In order to assess the degree of the cognitive impairment, the selected participants' cognitive function was accessed prior to the intervention with the robot. For this purpose, the Short Portable Mental Status Questionnaire was used.

A grounded theory approach was used in order to analyze the collected data. It was of the authors' concern that a set of human and physical conditions were met in order to keep the patients' dignity. A set of three questions was systematically used to assess patients' memory and behavior: "Do you remember him?", "What is his name?", "Would you like to pet him?" All moments were video-recorded. When viewed, interviews were transcribed and the behaviors codified according to the most common patterns ("smiling", "motor activity" and "spontaneous speech").

The total number of participants was six elderly patients who lived at the same institution, either on a daily basis (4 participants) or just during the week (2 participants). For one week, each participant interacted individually with Furby ${ }^{\odot}$, on a daily basis. After this week, there was a time gap of four days where participants did not interact and did not have any mention of Furby ${ }^{\odot}$. On the fifth day, participants were exposed to Furby ${ }^{\odot}$ again, 
on an individual level. The time of the interaction with Furby $^{\odot}$ wasn't defined so as to best adapt to the patients' attention span. Written consent was requested from the institution where participants were living at, as well as from the patients and/or their legal representative. This observation was approved by the investigator's institutional review board.

Analyzing the findings, it was possible to see that Furby ${ }^{\odot}$ demonstrated to alter patients' behaviors (considering both patients with moderate and severe dementia). Taking into account that almost all participants smiled while interacting with the robot, this may be interpreted as a sign of their comfort and well-being. Interacting with Furby $^{\odot}$ also increased motor activity as elderly patients would move their hands and arms in order to comply to simple tasks such as stroking or holding it. Furthermore, it also facilitated that patients spoke spontaneously (by telling it stories and asking it questions) and also promoted the interpretative thinking, as some participants tried to understand and translate into words some of the sounds produced by Furby ${ }^{\odot}$.

The findings also suggest that Furby ${ }^{\odot}$ also showed an improvement in the whole institution-from the third day on, other residents would stand up from their chairs and come closer to the participant whenever the interaction with Furby ${ }^{\odot}$ was taking place. We also recorded moments where elderly people would share emotions, comment and laugh whenever the robot was placed between them. Furthermore, some residents would even wave at Furby $^{\odot}$, asking for to come closer whenever they spotted it in the room.

We also observed that Furby ${ }^{\odot}$ spiked the curiosity of the healthcare professionals working at the institution. They showed intention of interacting with it, although most of them ended up describing it as "annoying".

As limitations of this observation, we cannot overlook the Hawthorne effect, term that is used to describe the change of behavior due to the attention that a participant is receiving [18]. Patients interacted both with the robot and the therapist for the first time, but thanks to the recordings, it was possible to focus our observation on the participants' interactions with the robot. By including a four day break, the researchers also wished to diminish the Hawthorne effect. Another limitation was regarding the time that was spent with the robot-in order not to alter and interfere with the patients' well-being, the authors had to adapt the time of interaction with Furby ${ }^{\odot}$ according to the patients' momentary state of mind. As such, it was not possible to establish an advisable time for exposition.

Furthermore, Furby ${ }^{\oplus}$ 's design also posed a limitation. Sometimes, it was difficult for patients to grasp and hold it as it's a relatively small robot (this was clearer in bed- ridden patients due to muscular atrophy). Furthermore, it was also difficult to control Furby ${ }^{\odot}$ 's sounds and personality. Furby ${ }^{\odot}$ has a rapidly switching personality, so the therapist needed to always pre-set it to its "happy" personality before giving it to the participant. Even so, this personality startled some of the participants as it included loud sounds and sudden movements with the ears and body.

This study wished to study how a widely commercialized zoomorphic robot, developed for children, may also be used as a therapeutic robot for elderly patients with dementia.

The findings suggest that Furby ${ }^{\odot}$ has some positive results in elderly patients with dementia, who are living at an institution. It has shown results both in patients, who were living as permanent residents and in those living in a day center setting, being a means to facilitate the expression of positive emotions as well as by stimulating motor and cognitive activity.

However, Furby ${ }^{\odot}$ has shown to have some limitations in terms of its design, namely in patients that were bedridden and in terms of controlling its personality and sounds.

Demographics are showing that the world's population is rapidly growing older, which consequently equals to a higher prevalence of no communicable diseases. Dementia is one of the diseases that is closely linked with ageing and where complementary therapies have shown to have an important role in terms of improving patients' well-being. As the application of zoomorphic robots is inspired in animal-assisted therapy, there is a strong possibility for it to improve and ease some symptoms related to dementia.

More research is needed to study the effects of social robots in elderly patients with dementia, comparing different models in order to develop the most adequate robot for this purpose. By providing a sense of companionship and consequently tackling the increasing social epidemic that is loneliness, it will be possible for healthcare systems to offer a solution to improve these patients' quality of life.

\section{References}

1. Robinson H, MacDonald BA, Kerse N, Broadbent E (2013) Suitability of healthcare robots for a dementia unit and suggested improvements. J Am Med Dir Assoc 14: 34-40.

2. Wu YH, Fassert C, Rigaud AS (2012) Designing robots for the elderly: appearance issue and beyond. Arch Gerontol Geriatr 54: 121-126.

3. Mordoch E, Osterreicher A, Guse L, Roger K, Thompson $G$ (2013) Use of social commitment robots in the care of elderly people with dementia: a literature review. Maturitas 74: 14-20. 
4. Shibata T, Wada K (2011) Robot Therapy: A New Approach for Mental Healthcare of the Elderly - a mini-review. Gerontology 57: 378-386.

5. WHO (2015) Ageing and health.

6. Wada K, Shibata T, Musha T, Kimura S (2008) Robot therapy for elders affected by dementia. IEEE Eng Med Biol Mag 27: 53-60.

7. Burton A (2013) Dolphins, dogs, and robot seals for the treatment of neurological disease. Lancet Neurol 12: 851852.

8. O'Haire M (2010) Companion animals and human health: Benefits, challenges, and the road ahead. J Vet Behav Clin Appl Res 5: 226-234.

9. Bernabei V, De Ronchi D, La Ferla T, Moretti F, Tonelli L, et al. (2013) Animal-assisted interventions for elderly patients affected by dementia or psychiatric disorders: a review. J Psychiatr Res 47: 762-773.

10. Šabanović S, Bennett CC, Chang WL, Huber L (2013) PARO robot affects diverse interaction modalities in group sensory therapy for older adults with dementia. IEEE Int Conf Rehabil Robot Proc 2013: 6650427.

11. Wada K, Takasawa Y, Shibata T (2013) Robot therapy at facilities for the elderly in Kanagawa prefecture - A report on the experimental result of the first week. IEEE ROMAN, Japan, 757-761.
12. Giusti L, Marti P (2006) Interpretative Dynamics in Human Robot Interaction. The 15th IEEE International Symposium on Robot and Human Interactive Communication ROMAN, Italy, 111-116.

13. Birks M, Bodak M, Barlas J, Harwood J, Pether M (2016) Robotic Seals as Therapeutic Tools in an Aged Care Facility: A Qualitative Study. J Aging Res 2016: 8569602.

14. Kawaguchi $Y$, Wada K, Okamoto M, Tsujii T, Shibata T, et al. (2012) Investigation of brain activity after interaction with seal robot measured by fNIRS. IEEE RO-MAN, Japan, 571-576.

15. Chang WL, Sabanovic S, Huber L (2013) Use of seal-like robot PARO in sensory group therapy for older adults with dementia. 8th ACM/IEEE International Conference on Human-Robot Interaction (HRI), USA 101-102.

16. Moyle W, Jones C, Cooke M, O'Dwyer S, Sung B, et al. (2013) Social robots helping people with dementia: Assessing efficacy of social robots in the nursing home environment. The 6th International Conference on Human System Interaction (HSI), Australia, 608-613.

17. https://en.wikipedia.org/w/index.php?title=Furby\&oldid $=730270535$

18. McCarney R, Warner J, lliffe S, van Haselen R, Griffin M, et al. (2007) The Hawthorne Effect: a randomised, controlled trial. BMC Med Res Methodol 7:30. 